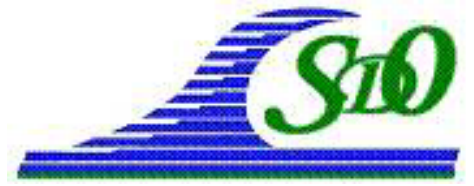

XI ìmes Journées Nationales Génie Côtier - Génie Civil

Les Sables d'Olonne, 22-25 juin 2010

DOI:10.5150/jngcgc.2010.059-L C Editions Paralia CFL

disponible en ligne - http://www.paralia.fr - available online

\title{
Cartographie de l'état du Domaine Public Maritime par télédétection très haute résolution : Application au Bassin d'Arcachon
}

\author{
Virginie LAFON $^{1}$, Julie MUGICA ${ }^{2}$, Cyril MALLET ${ }^{2}$
}

1. G.E.O. Transfert, UMR CNRS 5805 EPOC, Université Bordeaux 1, Avenue des Facultés, 33405 Talence cedex, France.

v.lafon@epoc.u-bordeauxl.fr

2. BRGM Aquitaine, Parc Technologique Europarc, 24 avenue Léonard de Vinci, 33600 Pessac,France.j.mugica@brgm.fr ; c.mallet@brgm.fr

\section{Résumé :}

La gestion environnementale du Bassin d'Arcachon nécessite de disposer d'outils de suivi opérationnels d'indicateurs de qualité des milieux. L'ostréiculture, activité économique dominante après le tourisme, est implantée sur le Domaine Public Maritime sous forme de concessions qui, lorsqu'elles sont en activité, sont bien entretenues. Cependant, il existe des friches ostréicoles qui perturbent potentiellement l'état du milieu. Les gestionnaires déduisent l'état des concessions de la connaissance de terrain ou par photo-interprétation. Nous présentons ici une méthode de quantification semiautomatisée de l'état des parcs, basée sur des images de télédétection très haute résolution $(50 \mathrm{~cm})$, qui peut être déployée de façon régulière et opérationnelle.

Le traitement proposé permet d'extraire automatiquement les contours des enrochements, substrats durs et exploitations ostréicoles des estrans du Bassin d'Arcachon. La géométrie et l'orientation des contours renseignent l'analyste de façon objective sur la présence et l'état des objets mis en évidence (tables, récifs sauvages ...). Ce traitement a été appliqué à des vues aériennes et satellite couvrant quatre sites du Bassin d'Arcachon ayant également fait l'objet d'une mission d'observation de terrain. La comparaison des moyens d'observation (télédétection / terrain) valide l'approche de cartographie mise au point. En effet, l'état de la surface (entretenu / en friche) est correctement cartographié dans plus de $93 \%$ des cas par télédétection aérienne. En outre, la superficie des zones qui se révèlent douteuses a été calculée pour chaque parcelle afin d'affecter à chacune d'entre elles un attribut d'état en suivant la nomenclature des gestionnaires du milieu. Dans $90 \%$ des cas, la carte obtenue par télédétection offre un diagnostic identique à celui déterminé in situ.

Ces attributs d'état enrichissent le cadastre et complètent les couches vectorisées issues du traitement d'image. La télédétection est donc, dans ce contexte et au vu de ces résultats chiffrés, un outil de gestion du domaine pertinent et efficace.

Mots-clés :

Télédétection - Cartographie - Domaine Public Maritime - Conchyliculture - Gestion 


\section{Introduction}

En application du Contrat de Projet Etat Région 2007-2013, l'Europe (FEDER), l'Etat, le Conseil Régional d'Aquitaine, les départements de la Gironde, des Landes et des Pyrénées-Atlantiques, le Syndicat Intercommunal du Bassin d'Arcachon (SIBA), le BRGM et l'ONF se sont associés pour poursuivre les actions de l'Observatoire de la Côte Aquitaine (OCA) qui furent initiées en 1996.

La tâche 6 de l'OCA inclut les opérations d'entretien du Domaine Public Maritime (DPM) du Bassin d'Arcachon, et la gestion de problèmes environnementaux liés par exemple aux friches ostréicoles, à l'état des ouvrages, et la présence d'éléments non souhaitables sur l'estran. Afin de fournir un support à certaines de ces opérations, la réalisation d'une cartographie de l'état du DPM est envisagée. Le BRGM a été saisi par le Comité de Suivi de la Mesure 9-4-1 du CPER 2007-2013 pour fournir un avis technique sur les orientations de ce programme. L'une des propositions de cet avis consiste à établir un état du DPM par télédétection. GEOTransfert (Université Bordeaux 1) en a testé la faisabilité.

Quoique encore peu développée dans cet objectif, la télédétection très haute résolution est un outil adapté au suivi des zones de production aquacole (ROUPIOZ et al., 2009). En effet, les cultures de bivalves, ont, sur les images de télédétection, une signature très particulière par rapport à leur environnement liée à la fois à la forme des exploitations, qui constituent des alignements, et à la texture des amas de coquilles.

Cette dernière caractéristique a été exploitée dans le développement d'une chaîne d'analyse d'image orientée vers la caractérisation de l'état des concessions ostréicoles du DPM du Bassin d'Arcachon. Deux jeux de données à très haute résolution spatiale (images aériennes et vues satellites) ont été exploités dans le triple objectif :

a) extraire les contours des objets repérables, distinguer les zones productives des friches ostréicoles (infrastructures abandonnées, massifs sauvages ...), proposer une carte des zones pour lesquelles des travaux de réhabilitation semblent nécessaires ;

b) valider l'analyse cartographique faite par télédétection à l'aide d'observations réalisées avec un GPS Différentiel ;

c) proposer une stratégie robuste pour le suivi de l'état du DPM.

\section{Méthode}

La méthode d'analyse (figure 1) procède en plusieurs étapes. La première utilise deux paramètres de texture extraits d'une matrice de co-occurrence (HARALICK, 1979) : la moyenne et la variance. Une image est générée par la soustraction de ces deux paramètres, qui est ensuite lissée (filtre adaptatif de LEE, 1980). Ces étapes réduisent considérablement le bruit sur l'image résultante. Cette dernière est segmentée pour produire des structures constituées de pixels spatialement connectés et de texture semblable (SLIMANI et al., 1986). La texture spécifique des amas de coquilles d'huitres induit la constitution de structures différenciées des substrats meubles environnants. Un 
opérateur ENVI génère des vecteurs superposés aux contours de ces structures, qui sont finalement superposés au cadastre ostréicole afin pour chaque parcelle :

a) de définir un indicateur d'exploitation (non exploité, culture sur table, culture à plat) ;

b) de définir un indicateur d'état (pourcentage de surface hétérogène dans la parcelle).

Ces deux indicateurs sont attribués par l'analyste (expert pour l'aide à la décision). Une reconnaissance de terrain a été réalisée sur quatre sites distincts du DPM du Bassin d'Arcachon afin de valider l'analyse faite à partir des données de télédétection.

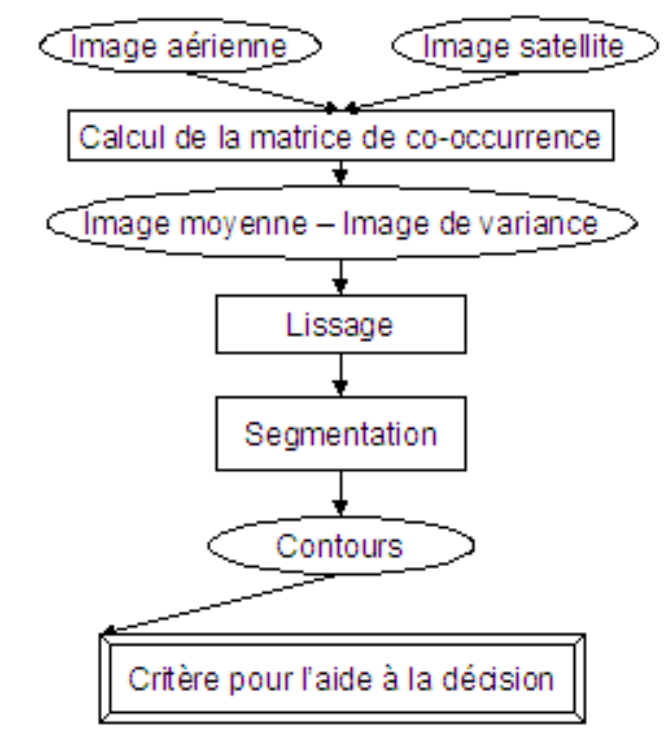

Figure 1. Organigramme de traitement.

\section{Données}

\subsection{Images aériennes}

Pour cette étude, nous avons utilisé des extraits des prises de vue verticales IGN (orthophographies) acquises le 27 août 2007 (propriété de l'IFREMER et de l'Observatoire de la Côte Aquitaine), géoréférencées et projetées dans le système Lambert II Etendu. La taille des pixels sur ces photographies est de $50 \mathrm{~cm}$.

\subsection{Imagerie WorldView-1}

Le satellite WorldView-1 (WV01) de DigitalGlobe acquiert des images en noir et blanc (panchromatique) d'une résolution de $50 \mathrm{~cm}$ pour une emprise de $246,4 \mathrm{~km}^{2}$ au nadir. Une image acquise le 26 avril 2008 (10:56:39 TU a été utilisée dans le cadre de cette étude. Elle a été livrée (Spot Image) en projection UTM Zone 30 (Datum WGS 84).

\subsection{Cadastre ostréicole}

Le cadastre ostréicole a été mis à disposition par le service "Cultures Marines et Environnement" de la Direction Départementale des Affaires Maritimes. 


\subsection{Observations in situ}

Deux missions d'observation sur le terrain ont été utilisées dans le cadre de cette étude. La première exécutée et traitée par le service "Cultures Marines et Environnement" de la Direction Départementale des Affaires Maritimes a été réalisée le 31 mars 2009. Cette mission concernait l'étude de l'état du banc de Mapouchet. Les surfaces qui doivent faire l'objet d'un nettoyage ont été identifiées et repérées, puis cartographiées. La seconde a été exécutée par les Affaires Maritimes, le BRGM et GEO Transfert le 8 juin 2009. A cette occasion, trois autres zones ont été visitées : le secteur de la Réousse, un estey du banc des Grahudes et une portion du secteur Haut Courbey / Canelon. Au total, 44 observations DGPS ont été collectées sur ces trois sites. La présence, le type de culture ainsi que l'état de surface ont été systématiquement renseignés.

\section{Résultats}

\subsection{Adéquation des supports image pour la cartographie du DPM}

Les deux types de support image ont été testés sur le site de la Réousse. Les autres sites ne permettent pas l'utilisation de l'imagerie WV01 car, situés en bord de chenal, ils sont affectés par le niveau d'eau, un peu élevé, un peu plus de 2 heures avant la basse mer. Le résultat graphique du traitement de l'orthophotographie est présenté sur la figure 2. Les contours des surfaces couvertes de coquilles d'huîtres résultant du traitement de l'orthophotographie sont reportés en blanc. La présence, la régularité et l'homogénéité, des contours (linéarité, symétrie des polygones) aident à qualifier les zones en bon état. $\mathrm{Au}$ contraire, les zones en friche sont délimitées par des contours sinueux (hétérogènes). Le résultat de la comparaison entre les observations de terrain et l'expertise menée à partir des images traitées sont détaillées dans le tableau 1. Sur 20 observations, les images WV01 donnent une interprétation incorrecte de l'état de la surface dans trois cas de zone en friche (soit un pourcentage de cohérence de $85 \%$ ). Les tables sont repérées dans 2 cas sur 8 , les cultures à plat, dans 3 cas sur 8 .

Les orthophotographies permettent d'augmenter la fiabilité de la détection du mode de culture et de l'état du DPM, certainement grâce à la richesse spectrale introduite par les bandes RVB. Dans ce cas, l'analyse est correcte à 95\% (1 seul cas est mal interprété). Les cultures à plat sont reconnues dans 5 cas sur 8 . Les cultures sur tables sont toujours perçues. Le support orthophotographique semble donc être le mieux adapté que l'imagerie WordView-1 pour la cartographie et l'analyse de l'état du DPM.

\subsection{Bilan de la détection de l'état du DPM par analyse d'orthophotographies}

Sur les secteurs des Grahudes et Haut Courbey/Canelon, 24 observations DGPS ont été effectuées. L'état, le type de culture et le degré d'entretien ont été renseignés sur chacun des sites. La comparaison de l'analyse des orthophographies traitées avec les observations in situ, montre un score proche de celui obtenu pour le secteur de la 
Réousse. En compilant l'ensemble des observations (44 au total), l'état de la surface révélé par l'analyse en segmentation est validé dans plus de $93 \%$ des cas par les observations de terrain. Les cultures à plat sont reconnues dans 5 cas sur 8 , les tables (exploitées ou non) dans 22 cas sur 24 .

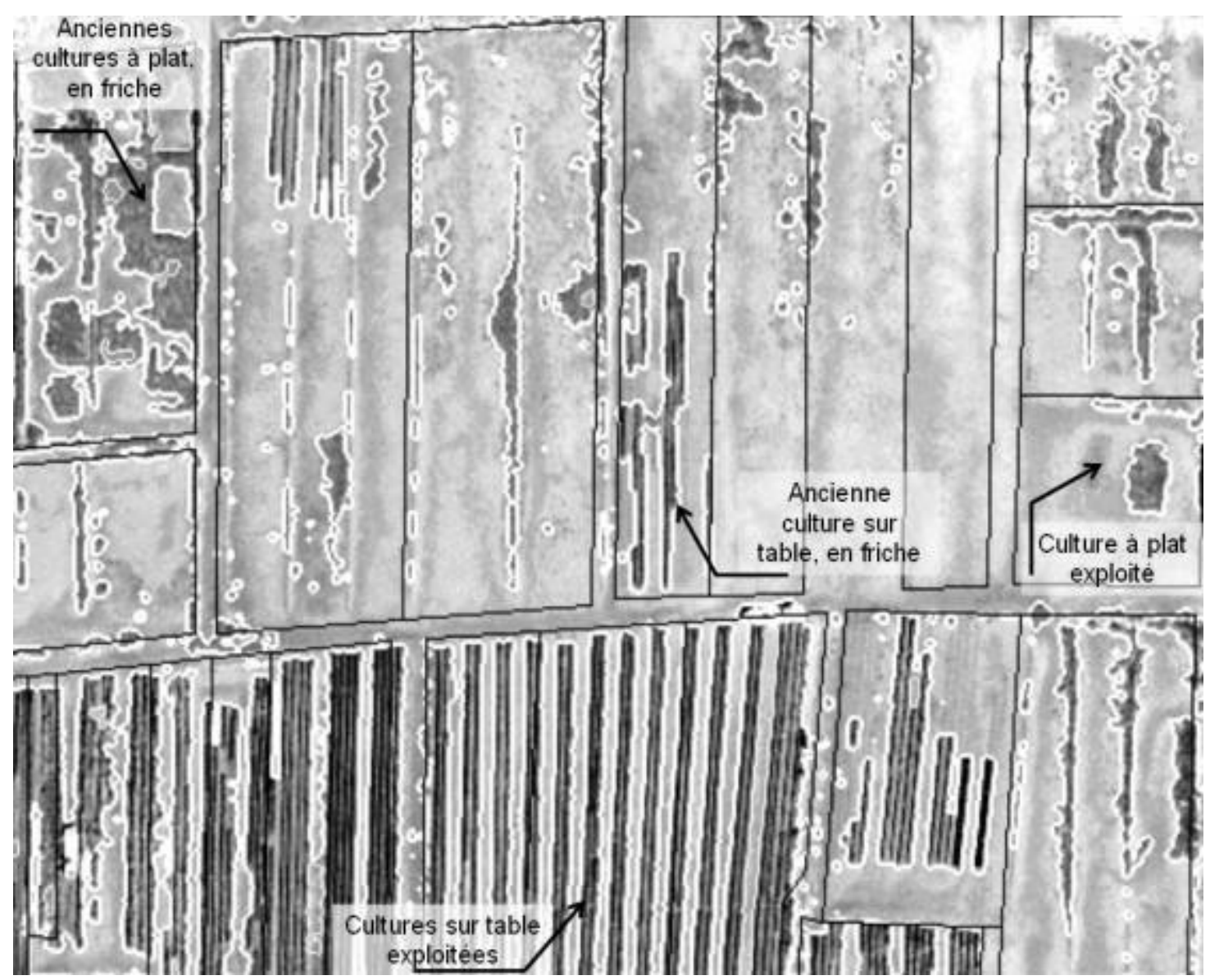

Figure 2. Orthophotographie du secteur de la Réousse. Les concessions sont encadrées de noir. Les vecteurs extraits du traitement sont en blanc.

\subsection{Approche cartographique pour l'aide à la décision}

Nous avons ici utilisé les levés de terrain effectués sur le banc de Mapouchet. Un polygone y a été généré afin de mettre en évidence l'ensemble des zones à nettoyer. Celles-ci représentent une superficie totale de 2,8 ha, dont près de 2,1 ha se situent dans le domaine concédé. Au total, sur 52 parcelles, 16 concessions sont signalées en friche, la friche recouvrant au moins $10 \%$ de leur superficie totale.

A partir de l'orthophographie traitée, la superficie totale des zones hétérogènes sur le banc de Mapouchet est de 3,7 ha (soit 20\% supérieure à celle déterminée sur le terrain). La surface révélée douteuse par analyse d'images recoupe 2,1 ha de la surface cartographiée in situ. Au total, 17 concessions présentent des hétérogénéités de surface 
sur plus de $10 \%$ de leur superficie, 14 d'entre elles correspondent aux observations in situ (encadré rouge et jaune figure 3).

Tableau 1. Comparaison entre les observations in situ et l'analyse faite à partir des supports WorldView et orthophotographie. La teinte gris clair indique une erreur d'analyse concernant la présence et/ou le mode d'exploitation, la teinte gris foncée, une erreur d'attribution concernant l'état de surface et éventuellement le mode de culture.

\begin{tabular}{|c|c|c|}
\hline Observation in situ & WorldView & Orthophotographie \\
\hline Non exploité - Entretenu & Non exploité - Homogène & Non exploité - Homogène \\
\hline Anciennes tables - En friche & Tables - Hétérogène & Tables - Hétérogène \\
\hline Limite d'une ancienne culture sur & Limite ouest d'une culture sur & Limite ouest d'une culture \\
\hline Table non entretenue - En friche & Table - Très Hétérogène & sur tTble - Très Hétérogène \\
\hline Non exploité - Entretenu & Non exploité - Homogène & Non exploité - Homogène \\
\hline Culture à plat - En friche & Non exploité - Homogène & Non exploité - Hétérogène \\
\hline Ancienne culture à plat - En friche & Non exploité - Homogène & Non exploité - Homogène \\
\hline Non exploité - Entretenu & Non exploité - Homogène & Non exploité - Homogène \\
\hline Non exploité - Entretenu & Non exploité - Homogène & Non exploité - Homogène \\
\hline Culture à plat - Entretenu & Non exploité - Homogène & Culture à plat - Homogène \\
\hline Culture à plat - Entretenu & Culture à plat - Homogène & Culture à plat - Homogène \\
\hline Anciennes tables - Entretenu & Non exploité - Homogène & Tables - Homogène \\
\hline Cultures à plat - En friche & Cultures à plat - Hétérogène & Cultures à plat - Hétérogène \\
\hline Cultures à plat - En friche & Cultures à plat- Homogène & Cultures à plat - Hétérogène \\
\hline Culture à plat - Entretenu & Culture à plat - Propre & Culture à plat-Propre \\
\hline Cultures sur tables - Entretenu & Non exploité - Homogène & Tables - Homogène \\
\hline Cultures sur tables - Entretenu & Non exploité - Homogène & Tables - Homogène \\
\hline Cultures sur tables - Entretenu & Non exploité - Homogène & Tables - Homogène \\
\hline Cultures sur tables - Entretenu & Non exploité - Homogène & Tables - Homogène \\
\hline Culture à plat - Entretenu & Non exploité - Homogène & Tables - Homogène \\
\hline Cultures sur tables - Entretenu & Non exploité - Homogène & Tables - Homogène \\
\hline
\end{tabular}

Deux des concessions signalées en friche à partir des observations de terrain ne sont pas repérées par le traitement d'image (encadré vert et jaune, figure 3). La première située tout au nord du banc montre des tables submergées. Le traitement ne produit aucun contour. Ainsi, le niveau d'eau limite l'applicabilité du traitement et empêche une analyse correcte de la concession. Pour la seconde concession, ni le traitement ni l'expertise ne mettent en évidence la dégradation de la surface qui a pourtant été mise en évidence sur le terrain. Des travaux de réhabilitation ont peut-être été entrepris aux cours des 11 mois séparant la prise de vue de l'inspection menée sur le terrain. 
Enfin trois concessions sont signalées en friche uniquement à partir du traitement de l'image. Pour deux d'entres elles, ni les résultats du traitement, ni l'expertise ne peuvent être mises en doute : les exploitations sont couvertes de coquilles. La cartographie de terrain ne les révèle pas, mais les levés à pied au cours d'une marée manquent peut-être d'exhaustivité. Pour la troisième concession, l'expertise est un peu plus discutable. Plusieurs contours irréguliers apparaissent dans la concession. Toutefois la couleur semble indiquer la présence de végétation. Malgré ces différences notées entre les cartes issues de l'analyse des orthophotographies et de la mission de terrain, plus de $90 \%$ des parcelles (47 sur 52) portent des attributs identiques.

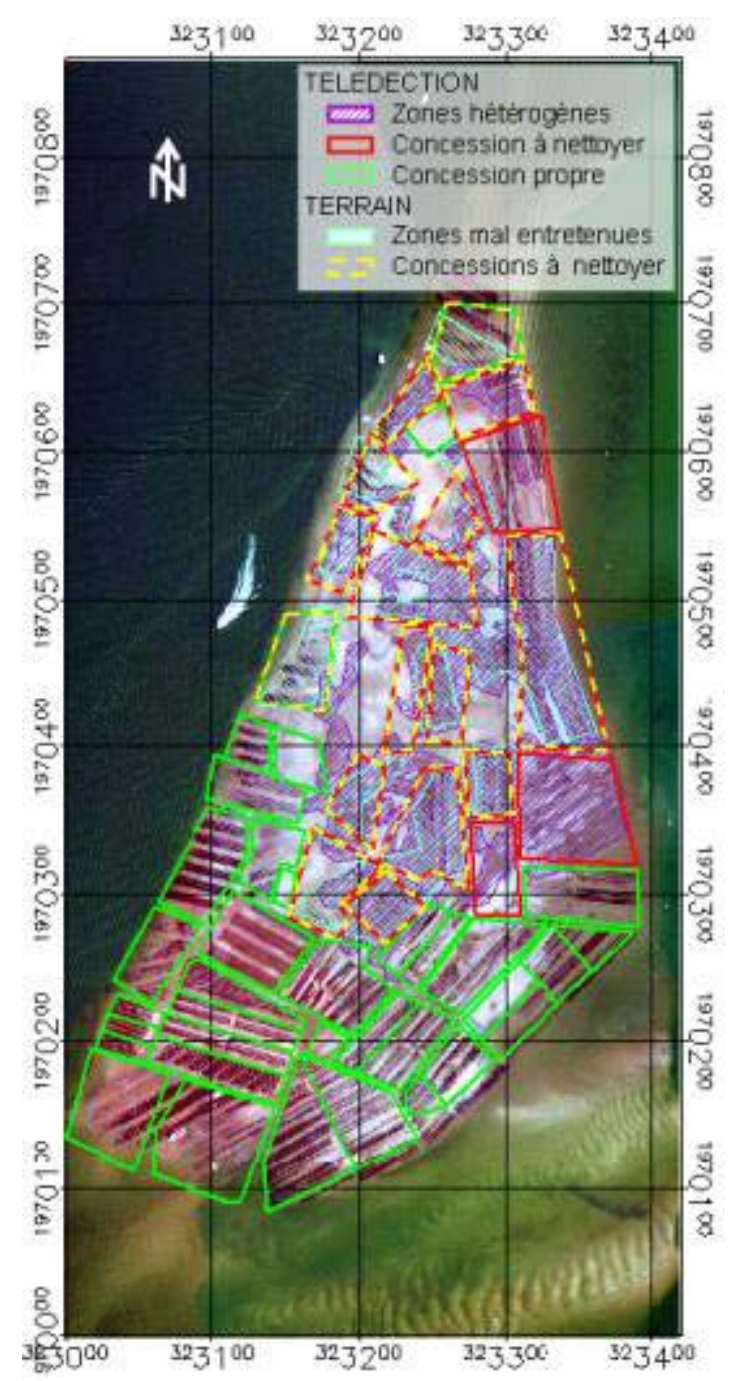

Figure 3. Cartographie in situ et par analyse d'orthophographie de l'état du DPM.

\section{Discussion et conclusions}

Ces travaux montrent l'utilité de la télédétection très haute résolution pour la cartographie de l'état du DPM dans le cas du Bassin d'Arcachon, où le domaine est en 
grande partie occupé par des concessions ostréicoles. Le gestionnaire du domaine doit en constater annuellement l'état d'entretien, ce qui entraîne d'importants frais de mission sur le terrain. Une méthode de traitement d'image, bien adaptée à l'usage d'orthophotographies d'une résolution de $50 \mathrm{~cm}$ a été développée et validée par des observations DGPS. Cette méthode, basée sur un analyse de texture permet de générer des contours des surface couvertes de coquilles d'huître. La présence et la forme de ces contours est une aide précieuse à la décision, car elles permettent de détecter dans de nombreux cas le mode de culture ainsi que l'état d'entretien de celles-ci. Ainsi l'état de surface (entretenu / en friche) est révélé dans plus de 93\% des cas. Une carte a été générée pour un banc couvert d'exploitations et comparée à une carte déduite d'observations sur le terrain. Les concessions portent des attributs identiques dans plus de $90 \%$ des cas. L'utilisation des orthophotographies est limitée par le niveau d'eau lié à la marée. Il est nécessaire d'acquérir les prises de vue à marée basse, de préférence en période de fort coefficient. Enfin, pour parfaire la qualité des cartes extraites des orthophotographies traitées, il faudra ajouter à l'analyse de la rugosité une analyse spectrale (qui tienne compte de la couleur) afin, le cas échéant, de ne pas confondre des zones d'herbier avec un couvert de coquilles. Les zones d'herbiers dans les parcs sont toutefois très peu fréquentes et restent un problème sporadique.

\section{Remerciements}

Nous remercions le service "Cultures Marines et Environnement" de la Direction Départementale des Affaires Maritimes de la Gironde, pour leur coopération et leur disponibilité sur le terrain.

\section{Références bibliographiques}

HARALICK R.M. (1979). Statistical and structural approaches to texture. Proceedings of the IEEE, vol. 67, No 5.

LEE J.-S. (1980). Digital Image Enhancement and Noise Filtering by Use of Local Statistics, IEEE Transactions on Pattern Analysis and Machine Intelligence, Vol PAMI2, No. 2, pp 165-168. doi:10.1109/TPAMI.1980.4766994

ROUPIOZ L., SCHMIDT A., KRAMER H., FEY F., CREMER J., DIJKMAN E., JANSEN J., DANKER N. (2009). Use of Remote Sensing methods in monitoring mussel beds in the Wadden Sea. 4th EARSeL Workshop on Remote Sensing of the Coastal Zone.

SLIMANI M., ROUX C., HILLION A. (1986). Image segmentation by cluster analysis of high resolution textured SPOT images. Architecture and Algorithms for Digital Image Proccssing, Proc. SPIE, vol. 596, pp 112- 119. 